\title{
Correlation between Alcohol-Based Handrub Consumption and Adherence to Hand Hygiene Protocols in Individual Nurses
}

\section{Yoko Yamamoto ${ }^{1}$, Kiyomi Harada ${ }^{1}$, Masako Murota ${ }^{1}$, Yukie Takishita ${ }^{1}$, Yoko Iwawaki ${ }^{1}$, Tomoko Matsuoka $^{1}$, Yukari Nishiuchi ${ }^{2}$, Toshie Ibayashi $^{2}$ and Kenya Matsumoto ${ }^{3}$}

${ }^{1}$ School of Nursing, Kyoto Prefectural University of Medicine, Japan

${ }^{2}$ Division of Nursing, University Hospital, Kyoto Prefectural University of Medicine, Japan

${ }^{3}$ School of Nursing, Kyoto Tachibana University, Japan

*Corresponding author: Yoko Yamamoto, School of Nursing, Kyoto Prefectural University of Medicine, 410 Nakagoryocho, Kamigyo-ku, Kyoto 602-0857, Japan, Tel: +81-75-212-5433, ORCID iD: 0000-0002-8699-7213

\begin{abstract}
Background: Hand hygiene helps prevent health careassociated infections. Prior studies have investigated methods to measure adherence to hand hygiene protocols and have attempted to determine the correlation between alcohol-based handrub consumption and hand hygiene adherence via direct observation in healthcare facilities or wards, but there remains need for methods measuring hand hygiene adherence in individual health care workers. The failure of even one worker to adhere to the hand hygiene protocols can increase the risk of infection. We aimed to examine the correlation between alcohol-based handrub consumption and adherence to hand hygiene protocols by direct observation in individual nurses.

Methods: We surveyed regarding alcohol-based handrub consumption and directly observed 17 nurses engage in hand hygiene practices at the University hospital in Japan.

Results: There was a significant, positive and moderate correlation between alcohol-based handrub consumption among day shift nurses ( $\mathrm{mL} /$ patient-day) and the rate of adherence to hand hygiene protocols (\%) on direct observation $[r=0.543(p=0.045)]$

Conclusion: Alcohol-based handrub consumption per patient-day during the day shift can be used to validate direct observation compliance rates of hand hygiene activities among individual nurses.
\end{abstract}

\section{Keywords}

Hand hygiene, Alcohol-based handrub consumption, Direct observation, Correlation

\section{Abbreviations}

ICU: Intensive Care Unit; WHO: World Health Organization

\section{Introduction}

Hand hygiene is an important measure for preventing healthcare-associated infections. Among other things, a nurse's hand hygiene is important in terms of duties that mostly involve frequent contact with patients. Several studies have investigated methods to measure adherence to hand hygiene protocols. Such measures are routinely performed in healthcare facilities or wards. However, there is an unmet need for methods that measure adherence to hand hygiene protocols in individual health care workers, because the failure of even one worker to adhere to the hand hygiene protocols can increase the risk of infection. Although direct observation is the gold standard and the only way to reliably capture all hand hygiene activities, direct observation by expert observers is time consuming and labour intensive [1]. Measurable use of hygiene products is simpler to obtain, but it is a relatively objective method. In general, the measurable use of alcohol-based handrub is calculated per 1 or 1,000 patient-days, whereas, by direct observation, the rate of adherence to hand hygiene protocols is calculated as the number of hand hygiene activities divided by number of hand hygiene opportunities, as defined by 'My 5 Moments for Hand Hygiene' according to the World Health Organization (WHO) guidelines [1]. However, when we calculate alcohol-based handrub consumption, if hand hygiene opportunities are not measured as per patient-day, our results

Citation: Yamamoto Y, Harada K, Murota M, Takishita Y, Iwawaki Y, et al. (2018) Correlation between Alcohol-Based Handrub Consumption and Adherence to Hand Hygiene Protocols in Individual Nurses. Int Arch Nurs Health Care 4:111. doi.org/10.23937/2469-5823/1510111

Accepted: November 28, 2018: Published: November 30, 2018

Copyright: (c) 2018 Yamamoto Y, et al. This is an open-access article distributed under the terms of the Creative Commons Attribution License, which permits unrestricted use, distribution, and reproduction in any medium, provided the original author and source are credited. 
will not agree with measures of adherence via direct observation. In addition, adherence to hand hygiene protocols by handwashing may not reflect the volume of alcohol-based handrub consumption. Conversely, implementation of hand hygiene at a situation that does not fall under 'My 5 Moments for Hand Hygiene' increases the alcohol-based handrub consumption.

Prior studies have attempted to determine the correlation between alcohol-based handrub consumption and adherence to hand hygiene protocols via direct observation in healthcare facilities or wards. Most reports showed no correlation [2-6], with only one study showing a correlation [7]. In addition, there are no studies that focus on this adherence among individual healthcare workers. Therefore, the purpose of this study was to examine, via direct observation, the potential correlation between alcohol-based handrub consumption and the adherence to hand hygiene protocols in individual nurses.

\section{Methods}

The study participants were nurses working in general wards (medical, surgical and paediatrics) at the University hospital in Japan. Each nurse had a nursing employment history of fewer than three years. We surveyed alcohol-based handrub consumption in individual nurses from October 2017 to November 2017. The nurses carried their alcohol-based handrub bottles (Sanisara ${ }^{\circledR}$ W, NXT 250 mL model; Saraya Corporation) with them while working and reported when the bottles were opened. First, we recorded the opening date of alcohol-based handrub bottles as reported by the nurses during the investigation period. Further, we examined the working shift of the nurses during that period and the number of patients who were being cared for by the nurses during the shift.

A researcher trained in infection control protocols performed direct observation of hand hygiene activities. These open observations were performed over the course of 10 opportunities for hand hygiene activities per participant during weekday day shifts (from 08:00 to $16: 45)$. Each opportunity was defined as per 'My 5 Moments for Hand Hygiene' from the WHO [1]. We observed the quality of hand hygiene activities as well as actual hand hygiene protocols as per the WHO guidelines (Handwashing: Covering all surfaces, duration and drying; Antiseptic handrub: Single-use amount, covering all surfaces, duration and drying). The rate of adherence to hand hygiene protocols (\%) was calculated as the number of hand hygiene activities divided by the number of hand hygiene opportunities times 100. Alcohol-based handrub consumption ( $\mathrm{mL} /$ patient-day) was calculated as $250 \mathrm{~mL} /$ the total number of patients on each nurse's caseload on that particular day. When calculating alcohol-based handrub consumption $(\mathrm{mL} /$ patient-day) during day shifts only, we chose one bottle $(250 \mathrm{~mL})$ that was used during the day shifts only, and when calculating the consumption ( $\mathrm{mL} /$ patient-day) during day plus night shifts, we chose one bottle (250 $\mathrm{mL}$ ) that was used during both day plus night shifts.

The SPSS 24.0 software (IBM, Tokyo, Japan) was used to conduct statistical analyses. We used Spearman rank correlation coefficients to generate correlations between alcohol-based handrub consumption $(\mathrm{mL} /$ patient-day) and the rate of adherence to hand hygiene protocols (\%). The statistical significance was 2 -sided with the significance level set at 0.05 .

The Institutional Review Board of the Kyoto Prefectural University of Medicine approved all protocols of this study (Notice number of determination: ERB-E-354-1, 2017).

\section{Results}

\section{Alcohol-based handrub consumption in individual nurses}

Seventeen nurses participated in this study. Their average duration of employment as a nurse was 1.8 years (range: 1.0-3.0). Of those, 12 calculated their alcohol-based handrub consumption during day plus night shifts, and 14 calculated day shift use only. During the day shift, the number of patients on each nurse's caseload was an average of 4.0 (range: 3.0-4.7) patients per day. The average alcohol-based handrub consumption in individual nurses was $9.1 \mathrm{~mL} /$ patientday (range: 3.4-17.9) during day plus night shifts and $16.4 \mathrm{~mL} /$ patient-day (range: 6.6-27.8) during day shift only.

\section{Rate of adherence to hand hygiene protocols determined by direct observation}

The average direct observation time per participant was 36.1 min (range 20-60). During this period, the alcohol-based handrub was used during $92.1 \%$ of the total observations and handwashing was completed on $7.9 \%$ of the total observations. The observed handwashing scenarios included prior to blood transfusion and infusion, after touching a patient who was with contact precautions, and after situations with high risk of body fluid exposure. The rate of adherence to incomplete hand hygiene protocols (in which an insufficient amount of alcohol-based handrub was used, there was an uncertain application of handrub to the hands, the hands were not rubbed for the recommended duration, or drying was incomplete) was $92.9 \%$ (range $70-100)$. The rate of adherence to complete hand hygiene protocols was $27.9 \%$ (range 0-80). Implementation of hand hygiene for situations that does not fall under 'My 5 Moments for Hand Hygiene' comprised $7.6 \%$ of the total observations.

\section{Correlation between alcohol-based handrub con- sumption and rate of adherence to hand hygiene protocols}

The correlation between alcohol-based handrub consumption during day plus night shifts $(\mathrm{mL} /$ patient- 


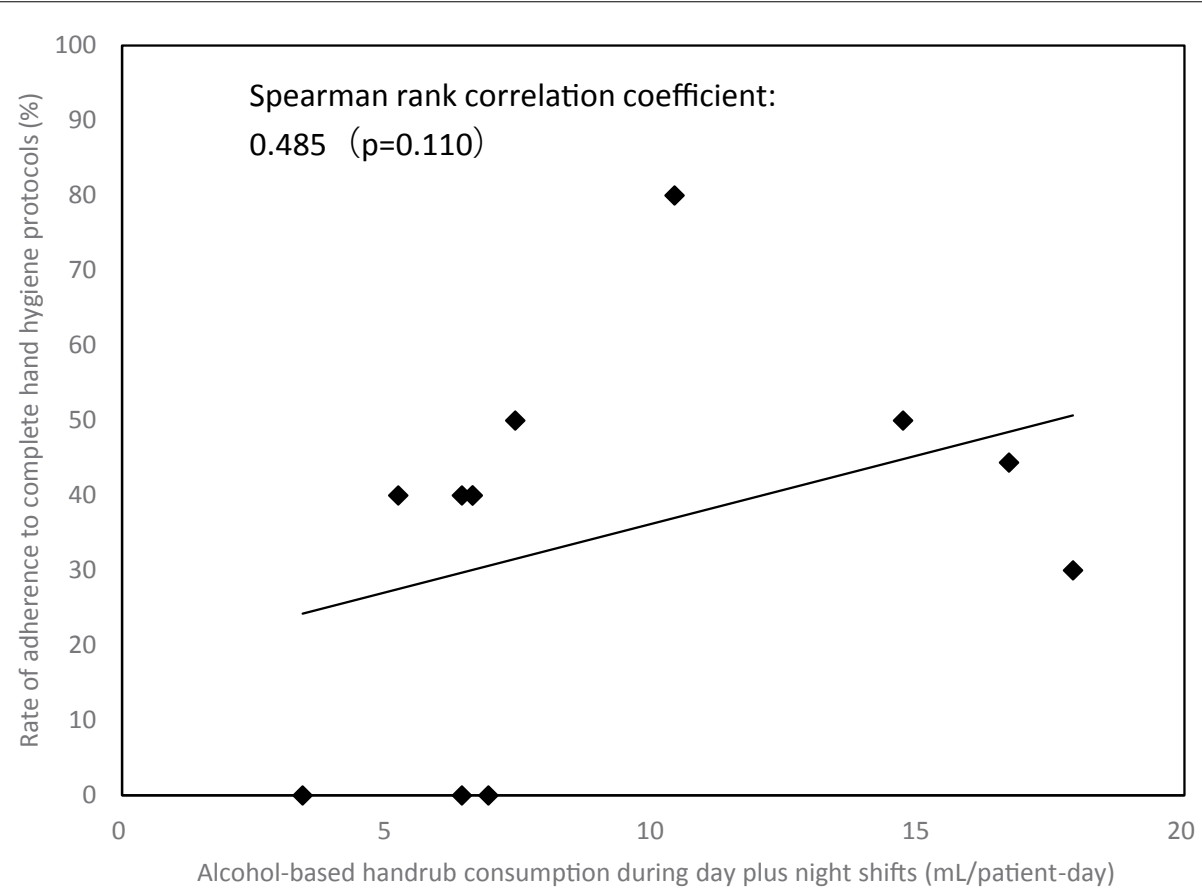

Figure 1: Relationship between alcohol-based handrub consumption during day plus night shifts (mL/patient-day) and rate of adherence to complete hand hygiene protocols (\%).

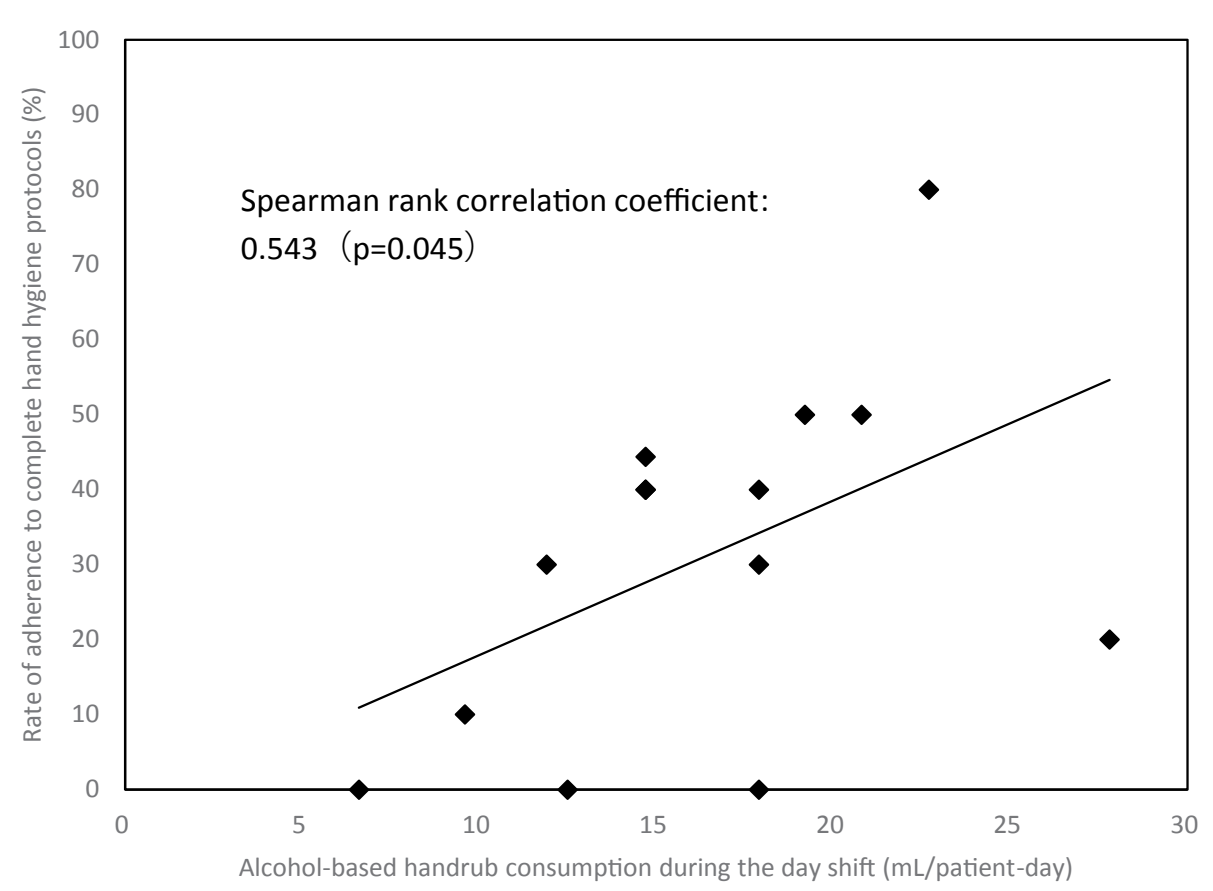

Figure 2: Relationship between alcohol-based handrub consumption during the day shift (mL/patient-day) and rate of adherence to complete hand hygiene protocols (\%).

day) and rate of adherence to complete hand hygiene protocols was $0.485(p=0.110)$ (Figure 1). When only the day shift was considered, the correlation was 0.543 $(p=0.045)$ (Figure 2).

\section{Discussion}

We found a positive, moderate correlation between alcohol-based handrub consumption per patient-day in nurses working the day shift and the rate of adherence to hand hygiene protocols, as measured via direct observation. Our results differed from those generated by previous studies [2-6]. This can be attributed to the number of opportunities for hand hygiene activities that were considered in the present study when calculating alcohol-based handrub consumption ( $\mathrm{mL} /$ patient-day). In other words, we considered that the opportunities for hand hygiene activities per patient were relatively similar as this study was conducted in general wards and all the nurses covered similar numbers of patients per shift. If we had conducted our investigation at a different location, such as the intensive care unit (ICU), we might have obtained a different result. In such a location, although each nurse cares for fewer patients, there is a large number of hand hygiene opportunities associated 
with caring for that patient. Therefore, although the compliance rate is low, there is a possibility that the amount of consumption will increase because of several opportunities. The same can be considered for the night shift situation. In the present study direct observations were not performed during the night shift; so that, it will be analysis assuming that the rate of adherence to hand hygiene protocols of individuals is the same even in the night shifts. It is likely that there are fewer opportunities for hand hygiene activities during the night shift; therefore, although the compliance rate is high, there is a possibility that the amount of consumption will decrease. Therefore, we believe that it is important to consider the number of hand hygiene opportunities while determining hand hygiene compliance based on alcohol-based handrub consumption.

In addition to opportunities, we considered the possibility of a mismatch between the alcohol-based handrub consumption and the rate of adherence to hand hygiene protocols when handwashing. Our results indicated that handwashing was performed in fewer than $10 \%$ of all the observations; therefore, we presume this was not a large mismatch factor. Under circumstances wherein antiseptic handrub was recommended as the first choice since 2002 [8], it is appropriate to use alcohol-based handrub consumption as an evaluation index. In addition, our observed rate of adherence to hand hygiene during a situation that does not fall under 'My 5 Moments for Hand Hygiene' was low (7.6\% of the total observations); therefore, we do not consider this to be an issue of major concern. More than anything, a major influence on our observed "complete" adherence rate was inappropriate antiseptic handrub method. Inappropriate antiseptic handrub method refers to less consumption, which has led to lower compliance rate. Therefore, considering the number of opportunities, consumption and compliance rate may be correlated.

This study had several limitations. First, the participants had relatively limited nursing experience $<$ 3 years) and were employed at a single facility; therefore, our findings may be difficult to generalise to nurses as a population. Second, there was a problem of validity related to having 10 direct observation opportunities per participant. Although the WHO recommends 200 observational opportunities per organisation, with approximately 20 minutes per observation, it does not give specific per-person guidelines [9]; therefore, further verification is necessary. Third, since the direct observations used in this study were not 'shadow observations', we cannot dismiss a potential influence from the Hawthorn effect. Finally, we recognise the problem of the reliability of alcohol-based handrub consumption per patient-day. When calculating alcoholbased handrub consumption ( $\mathrm{mL} /$ patient-day), we chose only one bottle each from the ones used during just the day shift or day plus night shifts, respectively.
In conclusion, we believe that alcohol-based hand rub consumption per patient-day during the day shift can be used to help validate direct observation compliance rates of hand hygiene activities among individual nurses. Although this index requires a plurality of data and it is a bit complicated to use as a daily evaluation, it can be used as an individual evaluation with objectivity similar to direct observation, albeit easier than direct observation. In the future, we would like to perform repeated examinations to determine the generalizability of our results. Future studies should also be performed in ICU settings or among night shift workers; furthermore, hand hygiene practices among individuals from other occupations should be examined.

\section{Acknowledgements}

We thank the Director and Deputy Director of nursing and the head nurses and other nurses who participated in this investigation. This study was conducted with support from the Kyoto Prefectural University of Medicine, Japan. We have no conflicts of interest to declare. Finally, we thank MARUZEN-YUSHODO for the English language review.

\section{References}

1. World Health Organization (2009) WHO Guidelines on hand hygiene in health care.

2. Scheithauer S, Haefner $H$, Schwanz T, Schulze-Steinen $\mathrm{H}$, Schiefer J, et al. (2009) Compliance with hand hygiene on surgical, medical, and neurologic intensive care units: Direct observation versus calculated disinfectant usage.Am J Infect Control 37: 835-841.

3. Marra AR, Moura DF, Paes AT, dos Santos OF, Edmond MB (2010) Measuring rates of hand hygiene adherence in the intensive care setting: A comparative study of direct observation, product usage, and electronic counting devices.Infect Control Hosp Epidemiol 31: 796-801.

4. Sroka S, Gastmeier P, Meyer E (2010) Impact of alcohol hand-rub use on meticillin-resistant Staphylococcus aureus: An analysis of the literature. J Hosp Infect 74: 204-211.

5. Morgan DJ, Pineles L, Shardell M, Young A, Ellingson K, et al. (2012) Automated hand hygiene count devices may better measure compliance than human observation. Am J Infect Control 40: 955-959.

6. Sodré da Costa LS, Neves VM, Marra AR, Sampaio CamargoTZ, Fátima dos Santos Cardoso MF, et al. (2013) Measuring hand hygiene compliance in a hematologyoncology unit: A comparative study of methodologies. Am J Infect Control 41: 997-1000.

7. Eckmanns T, Schwab F, Bessert J, Wettstein R, Behnke $M$, et al. (2006) Hand rub consumption and hand hygiene compliance are not indicators of pathogen transmission in intensive care units.J Hosp Infect 63: 406-411.

8. Boyce JM, Pittet D (2002) Guideline for hand hygiene in health-care settings: Recommendations of the healthcare infection control practices advisory committee and the HICPAC/SHEA/APIC/IDSA hand hygiene task force. Infection Control \& Hospital Epidemiology 23: S3-S40.

9. World Health Organization (2009) Hand hygiene technical reference manual: To be used by health-care workers, trainers and observers of hand hygiene practices. 